\title{
Stiffness of the substrate influences the phenotype of embryonic chicken cardiac myocytes
}

\author{
Piyush Bajaj, ${ }^{1,2 *}$ Xin Tang, ${ }^{2,3 *}$ Taher A. Saif, ${ }^{2,3}$ Rashid Bashir ${ }^{1,2,4}$ \\ ${ }^{1}$ Department of Bioengineering, University of Illinois at Urbana-Champaign, Urbana, Illinois 61801 \\ ${ }^{2}$ Micro and Nanotechnology Laboratory, University of Illinois at Urbana-Champaign, Urbana, Illinois 61801 \\ ${ }^{3}$ Department of Mechanical Science and Engineering, University of Illinois at Urbana-Champaign, Urbana, Illinois 61801 \\ ${ }^{4}$ Department of Electrical and Computer Engineering, University of Illinois at Urbana-Champaign, Urbana, Illinois 61801
}

Received 23 April 2010; revised 22 July 2010; accepted 2 August 2010

Published online 11 October 2010 in Wiley Online Library (wileyonlinelibrary.com). DOI: 10.1002/jbm.a.32951

\begin{abstract}
We examined the effect of substrate stiffness on the beating rate, force of contraction, and cytoskeletal structure of embryonic chicken cardiac myocytes by culturing them on laminin-coated polyacrylamide (PA) substrates. Cells cultured on PA substrates with elasticity comparable to that of the native myocardium ( $18 \mathrm{kPa}$ ) exhibited the highest beating rate during the first few days of culture. The initial beating rate of individual cells on all the substrates varied significantly but began to converge within 5 days. We also examined the focal adhesions (FAs) and cytoskeletal structure on different substrates via confocal microscopy and found a higher percentage of FAs on tissue culture (TC) plastic dishes compared with the softer PA gels. Furthermore, highly aligned sarcomeric striations were clearly visible on $18 \mathrm{kPa}, 50 \mathrm{kPa}$, and TC dish,
\end{abstract}

whereas cells on $1 \mathrm{kPa}$ only showed nonaligned diffused striations. The force of contraction on these substrates was measured using a micro-electromechanical system force sensor, which showed that the force of contraction for the cells on TC dishes $(F=71.30 \pm 6.38 \mathrm{nN})$ was significantly larger than those cultured on the 18-kPa PA gel ( $F=30.16 \pm 3.83 \mathrm{nN})$. This is most likely due to the formation of higher percentage of FAs on the TC dishes compared with fewer FAs on the softer gels. Our cumulative findings can have a significant impact on the design of 3D cardiac tissue engineered scaffolds. (C) 2010 Wiley Periodicals, Inc. J Biomed Mater Res Part A: 95A: 1261-1269, 2010.

Key Words: substrate stiffness, focal adhesions, beating force, embryonic chicken, cardiac myocytes

\section{INTRODUCTION}

Living cells can sense their physical microenvironment and respond to mechanical cues with changes in their morphology, migration, division, and gene expression. Previous studies have shown that mesenchymal stem cells can differentiate to specific lineages/fates based on the stiffness of the substrates. ${ }^{1}$ Neurons have been shown to display increased branched actin structures on soft brain-like substrates compared with stiff substrates, whereas glial cells (i.e., astrocytes) from the central nervous system grow better on rigid substrates than on flexible ones. ${ }^{2}$ Fibroblasts have been shown to adjust their intracellular cell stiffness by changing their cytoskeletal structure to match the stiffness of the substrate on which they are cultured. ${ }^{3}$ Moreover, when NIH/3T3 fibroblasts were cultured on a substrate with varying rigidity along the surface, they readily migrated from the soft side of the substrate to the rigid side, which is called "durotaxis." ${ }^{4}$ Myoblasts, which are the precursors of myotubes, differentiate optimally and show actomyosin striations only on substrates with stiffness typical of a normal muscle. ${ }^{5}$ Wang et al. $^{6}$ demonstrated that stiffness of the substrate can also dictate whether a cell will grow or undergo apoptosis. Nontransformed NIH/3T3 fibroblasts cultured on soft substrates showed a decrease in the rate of DNA synthesis and an increase in the rate of apoptosis compared with the same cells cultured on rigid substrates.

The stiffness of the substrate also plays an important role in the development and the normal functioning of the myocardium. ${ }^{7}$ On average, the heart beats about 72 times a minute, which roughly translates to about 2.5 billion beats over the human lifetime. During this period, the heart responds to a variety of mechanical stresses based on the physiological conditions of the individuals. These mechanical stresses can be increased as a result of heart disease. The stiffness of healthy myocardium lies between the soft brain cells and the relatively hard cartilage/bone cells., ${ }^{8,9}$ Previous studies have indicated that after myocardial infarction, scar formation begins in the area around infarction and increases the stiffness of the myocardium locally by several folds. ${ }^{10}$ As a result, the force of contraction decreases, thereby affecting the performance of the left ventricles as well. ${ }^{11}$

Additional Supporting Information may be found in the online version of this article.

* These authors contributed equally to this work.

Correspondence to: T. A. Saif; e-mail: saif@illinois.edu or R. Bashir; e-mail: rbashir@illinois.edu

Contract grant sponsors: U.S. Army Medical Research \& Materiel Command (USAMRMC), The Telemedicine \& Advanced Technology Research Center (TATRC); contract grant number: W81XWH0810701 
In this study, the beating rate, force of contraction, and cytoskeletal structure of embryonic chicken cardiac myocytes (ECCMs) were quantified using a micro-electromechanical system (MEMS) force sensor by plating them on laminin-coated polyacrylamide (PA) substrates with stiffnesses of $1-50 \mathrm{kPa}$ and tissue culture (TC) dishes. The beating rate of the cells cultured on the $18-\mathrm{kPa}$ PA gel was the highest during the first few days. However, by the fifth day, the cells showed similar beating rates on all the substrates. The cells cultured on the rigid substrates showed higher percentage of focal adhesions (FAs) compared with those cultured on the softer substrates. Highly aligned sarcomeric striations were visible on the stiff substrates, whereas cells on the soft substrate only showed nonaligned diffused striations. It was also found that cells on rigid TC dishes generated larger forces most likely by forming higher number of FAs compared with 18-kPa PA gel. Because the stiffness of the biomaterial/substrate dictates the behavior of the cells, understanding it better can result in improved therapies for many heart-related diseases and for engineered heart tissue.

\section{MATERIALS AND METHODS}

\section{PA gel preparation and calibration}

PA gels were prepared following the protocols described by Wang and Pelham. ${ }^{12}$ In brief, PA gels of increasing stiffness were obtained by changing the concentration of acrylamide solution (BioRad Laboratories, Hercules, CA) from 5 to $8 \%$ and the concentration of bis-acrylamide solution (BioRad Laboratories) from 0.04 to $1.2 \%$ in $10 \mathrm{mM}$ HEPES (Sigma, St. Louis, MO). These gels were then activated with $1 \mathrm{~m} M$ Sulfo-SANPAH (Thermo Scientific, Rockford, IL) and conjugated with $5 \mu \mathrm{g} / \mathrm{mL}$ of laminin (Sigma). To test the attachment of the protein as a function of substrate stiffness, fluorescently tagged bovine serum albumin (Invitrogen, Carlsbad, CA) was coated on the gel, and it was found that the protein concentration was independent of the stiffness (see Supporting Information Fig. S1). The coating concentration has also been shown to be independent of the stiffness of the gel with other proteins such as collagen I, collagen IV, laminin, and fibronectin. ${ }^{13}$

To mimic a wide range of mechanical microenvironments, gels with physiologically relevant stiffnesses were produced. $^{14,15}$ Soft PA gels with stiffness $1.05 \pm 0.17 \mathrm{kPa}$ were used to mimic mammary gland, brain, and breast tissue, which have physiological stiffness ranging from 0.1 to 2 $\mathrm{kPa}^{16}$ Intermediate hard gels with stiffness $18.31 \pm 0.19$ $\mathrm{kPa}$ were used to mimic chicken embryonic myocardium, which have physiological stiffness ranging from 9 to 20 $\mathrm{kPa}^{8}$ Hard gels with stiffness $50.68 \pm 0.92 \mathrm{kPa}$ were used to mimic cartilage and bone. ${ }^{8}$ TC dishes served as the control for the study (stiffness of TC $\sim 3 \mathrm{GPa}$ ).

An Asylum atomic force microscope with precalibrated silicon nitride tip was used to characterize the stiffness of PA gels in phosphate-buffered saline (PBS, pH 7.4; Invitrogen) solution. ${ }^{17-19}$

\section{Cardiac cell isolation and culture}

Cardiac cells were obtained from chicken embryos during the 32-35 Hamburger Hamilton stage, which is approximately 8 days after conception. ${ }^{20}$ After sterilizing the eggshell with $70 \%$ ethanol, the embryo was removed, placed in a petridish containing Puck's saline, and quickly decapitated. The heart was removed, rinsed twice in PBS solution to remove the blood, and incubated in a solution containing $0.05 \%$ trypsin/PBS (Invitrogen) for $\sim 15$ minutes at $37^{\circ} \mathrm{C}$. Following enzymatic digestion, the solution containing the heart was pipetted gently to dissociate the tissue. About 100,000 cells $/ \mathrm{cm}^{2}$ were seeded on the laminin-coated substrates and cultured in minimum essential medium with alpha modification (Sigma), supplemented with 10\% fetal bovine serum (Sigma) and antibiotics $(100 \mathrm{U} / \mathrm{ml}$ penicillin and $100 \mu \mathrm{g} / \mathrm{ml}$ streptomycin) (Invitrogen). During data acquisition, the gels and the culture dishes were kept on a heated microscope stage to maintain a temperature of $37^{\circ} \mathrm{C}$, and a tube releasing $5 \% \mathrm{CO}_{2}$ was kept over the dishes to maintain a physiologically relevant $\mathrm{pH}$.

\section{Immunofluorescence and imaging}

Cells cultured on the PA gels and TC dishes were immunolabeled on the second and the fifth day in the study. Cells were fixed with $4 \%$ paraformaldehyde (Electron Microscopy Sciences, Hatfield, PA) for $30 \mathrm{~min}$ and then permeabilized with $0.2 \%$ Triton X-100 (Sigma) for $15 \mathrm{~min}$. Cells were then blocked with Image-iT FX (Invitrogen) for $30 \mathrm{~min}$. They were incubated in monoclonal mouse anti-vinculin (Sigma) at a 1:200 dilution in PBS at $4{ }^{\circ} \mathrm{C}$ overnight. These cells were permeabilized again in $0.2 \%$ Triton X-100 for $15 \mathrm{~min}$ and incubated in fluorescein isothiocyanate-conjugated goat anti-mouse (BioRad Laboratories) at a 1:200 dilution in PBS at $37^{\circ} \mathrm{C}$ for $2 \mathrm{~h}$. Cells were then incubated for $2 \mathrm{~h}$ in $6.6 \mu \mathrm{M}$ rhodamine-phalloidin (Invitrogen) and finally in $500 \mathrm{n} M$ of DAPI (Invitrogen) for $5 \mathrm{~min}$ at $37^{\circ} \mathrm{C}$. The immunolabeled cells were mounted in ProLong gold (Invitrogen) antifade reagent to prevent photobleaching. The cell preparations were imaged by a Zeiss LSM 710 confocal scanning laser microscope at $40 \times$, NA 1.4 water immersion objective.

\section{Image processing and image analysis}

Images were processed using ImageJ version $1.42 \mathrm{q}^{21}$ Area of the cell was found by first creating a maximum z-projection from the confocal images and then thresholding it so that the entire cell area was covered. Any object smaller than $3 \mu \mathrm{m}$ in radius was eliminated using the software to get rid of any cellular debris and then the percentage of the area covered by the cells was computed by the software. This percentage was converted to an area in square microns. To find the percentage of FAs, one confocal section per image was used, which is approximately $0.50 \mu \mathrm{m}$ from the interface between the cell membrane and the coverslip. The images were thresholded, and a Watershed segmentation algorithm was applied to the images to see only the $\mathrm{FAs}^{22}$ ImageJ was also used to create fluorescence intensity line profiles over FAs of actin and vinculin images around 

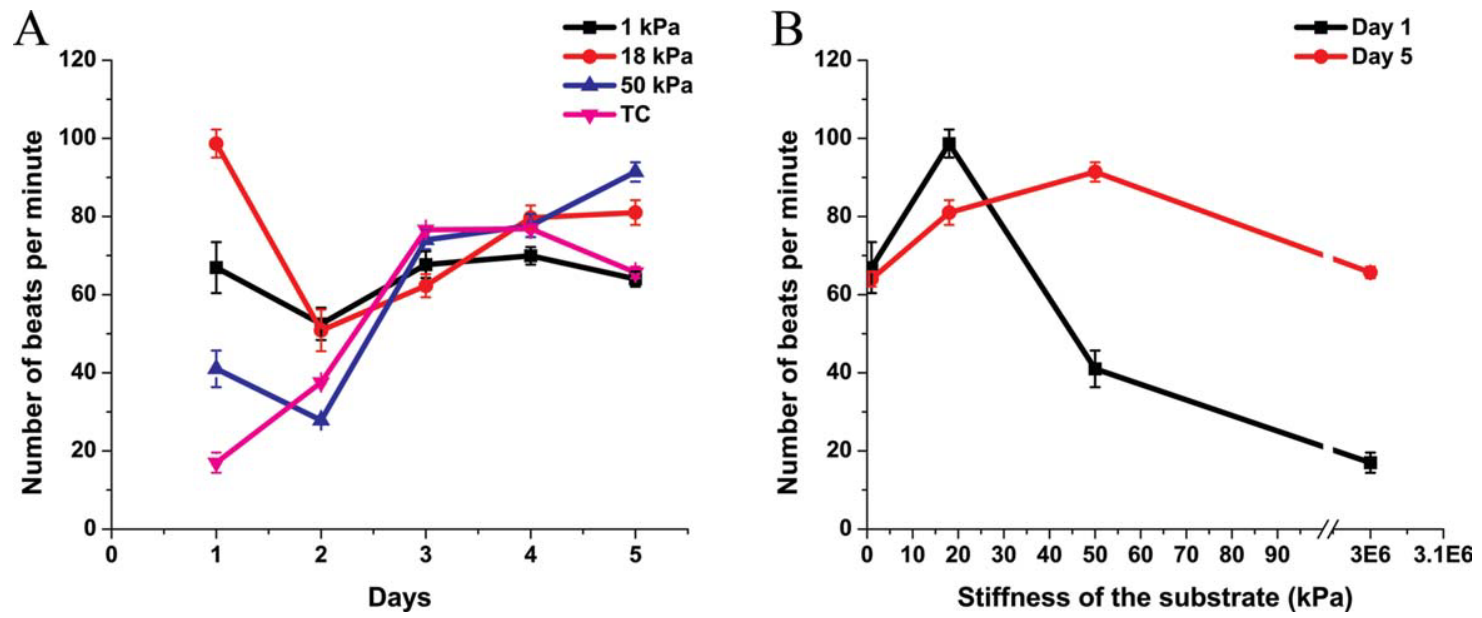

FIGURE 1. Beating rate of cardiac cells on the substrates (A). The beating rate of cardiac cells on $1 \mathrm{kPa}, 18 \mathrm{kPa}, 50 \mathrm{kPa}$, and TC dishes as a function of time over 5 days. (B) The beating rate of cardiac cells as a function of stiffness on the first and the fifth day of culture. Data shown are the mean $\pm \operatorname{SEM}(n=10)$.

different regions of the cell. The cells were found independently for computing the FA percentage.

\section{Statistical analysis}

Statistical analysis was performed using one-way ANOVA in OriginPro 8.1. Unless otherwise mentioned, the values reported are mean \pm standard error of the mean (SEM), and the results were considered statistically significant when the $p$ value was less than $0.05(p<0.05)$.

\section{RESULTS}

Beating rate of cardiac cells versus substrate rigidity ECCMs were seeded on laminin-coated PA gel substrates of varying stiffnesses, and the beating rate of the cells was monitored as a function of time over 5 days. Cells were independently chosen on all the substrates, and a 30-s video of the beating cells was taken every day for 5 days in culture. Figure 1(A) shows the variation in the average beating rate of the cardiac cells over this period. Note that the initial average beating rate varies significantly ranging from $<20$ beats per minute on the rigid TC dishes to 100 beats per minute on the $18-\mathrm{kPa}$ gel. However, the average beating rate converges to a more narrow range around $60-80$ beats per minute by the fifth day. Figure 1(B) shows the beating rate of the cardiac myocytes as a function of substrate stiffness after 1 and 5 days post-seeding. The 18-kPa substrate, which mimics the stiffness of the myocardium, shows the highest beating rate on the first day of culture. This beating profile on the first day can be approximated by a Gaussian curve for the stiffness of the substrate $(E)$. However, by the fifth day, the beating rate of the cells converge to a narrow range of $60-80$ beats per minute and no longer follows a bell curve.

\section{FA formation and cytoskeletal organization of cells on the different substrates}

Formation of FAs and the cytoskeletal organization of the cell were examined via confocal microscopy on the various substrates on the second and fifth day. FAs are specialized sites of adhesion developed by many cells in culture. They serve as the pivots to assist the intracellular pre-stress build-up and are the sites at which cell traction is transmitted to the substrate. They link the extracellular matrix components to intracellular cytoskeleton (actin filaments) via integrin receptors. FAs can be thought of as a sensory organ capable of responding to a variety of diverse external features such as ligand density, ${ }^{15,23}$ topography, ${ }^{24}$ and rigidity. $^{25,26}$ FAs are composed of many different types of proteins, such as paxillin, vinculin, and talin. ${ }^{27}$ However, it has been proposed that vinculin depletion leads to drastic changes in the motility of cells and FA sizes. ${ }^{28-30}$ Furthermore, vinculin is the most abundant FA protein. ${ }^{31}$ Hence, the cells on these substrates were labeled with anti-vinculin antibody.

The formation of FAs is a highly orchestrated event starting from: (1) Rho-stimulated contractility, (2) the generation of isometric tension in adherent cells, (3) bundling of actin filaments, (4) aggregation of integrins, and (5) activation of the focal adhesion kinase. ${ }^{32}$ To produce a more visual illustration of the relative intensity of actin and vinculin, fluorescence intensities in 1D line profiles drawn over FAs was compared and is shown in Figure 2. It can be seen from the line profile that the intensity of actin and vinculin present on the soft (1 $\mathrm{kPa}$ ) gel is small compared with the other substrates. The cells are not able to form many FAs because there is not enough actomyosin-based contractions on the soft substrate. ${ }^{8}$ Because one of the first events in the formation of FAs is reduced on the soft gel, other downstream events are also hindered and hence few FAs form on this substrate. This is consistent with previously published results that a smaller force is required to peel off cells from soft gels compared with the rigid glass. ${ }^{5}$ FA formation on this soft substrate occurs by nonspecific tyrosine hyperphosphorylation. ${ }^{8}$ On the other hand, the pixel intensity values for actin and vinculin is high on the stiff $(50 \mathrm{kPa})$ gel and the rigid (TC dish) substrate. Furthermore, there is 

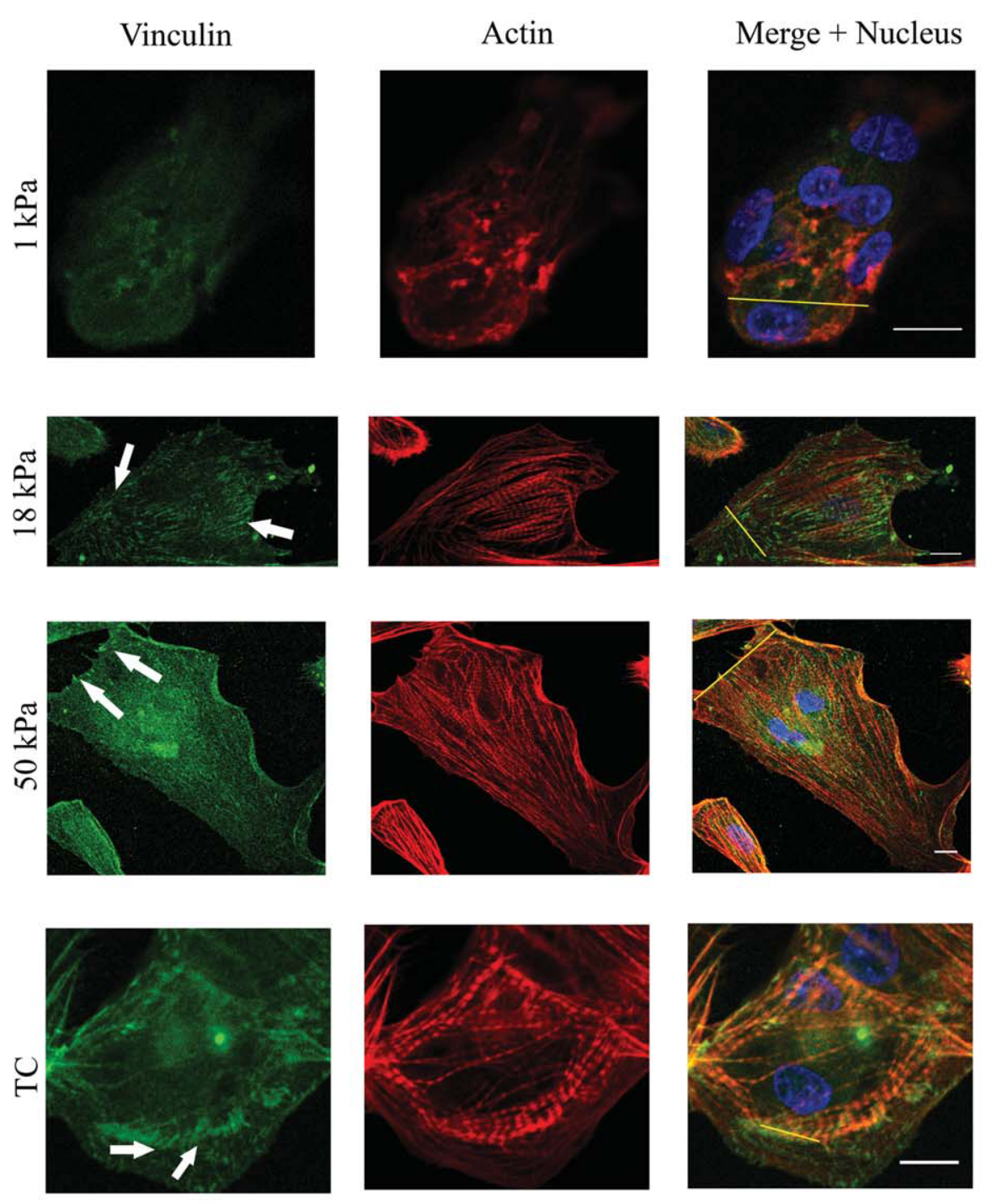
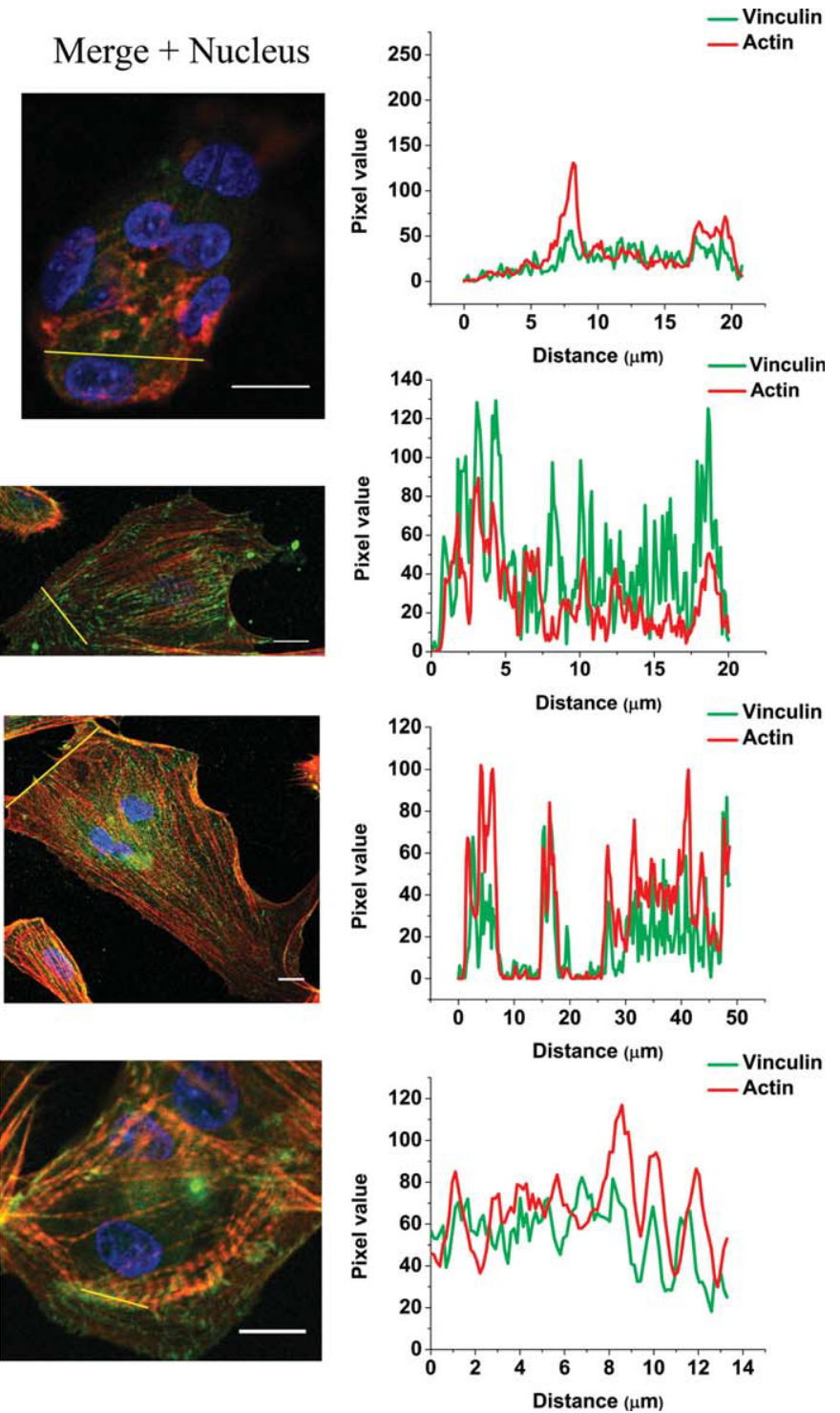

FIGURE 2. Relative fluorescent intensity of actin and vinculin for cells on the substrates. Sections of cardiac cells expressing vinculin and actin proteins on the different substrates are shown. Fluorescence intensity profiles depict the area of the line drawn in the merged images. The arrowheads show well-defined mature FAs. Scale bar: $10 \mu \mathrm{m}$.

colocalization of actin and vinculin, which leads to aggregation of integrins at the FA sites. ${ }^{30}$ On these stiff surfaces, FA development is possible by enhanced tyrosine phosphorylation on multiple FA proteins. ${ }^{33}$ In addition, a higher degree of organized cytoskeleton emerges with the increase in stiffness as shown in Figure 2. On the soft 1-kPa substrates, formation of tissue-like aggregates can be seen and only small nascent FAs are visible, whereas on the other substrates, cells acquire a more spread morphology and show welldefined prominent FAs of larger size.

Figure 3(A) shows a representative image of the FAs present on the cell and its corresponding area. Figure 3(B) shows that the percentage of FA increases as a function of substrate stiffness. Both the fibroblasts and the cardiac cells show higher percentage of FAs on the rigid (TC) substrate compared with the soft (1 kPa) PA gel and the 18-kPa gel.
Small nascent FAs, also referred to as focal complexes (FXs), are formed on soft 1-kPa substrates. ${ }^{34}$ These small FAs cannot provide the necessary adhesion to propel the cell forward. As a result, these FAs quickly disassemble, and the cells on the soft 1-kPa gel acquire a tissue-like morphology, where a lot of cells are present in the aggregate. However, on the comparatively stiffer substrates, small FAs quickly mature and form larger FAs, providing the necessary traction forces for the cells to acquire a more spread morphology. ${ }^{33,35,36}$ There is not a statistical difference in the percentage of FAs present on fibroblasts $(p=0.49)$ and cardiac cells $(p=0.95)$ for the two different days, suggesting that after the first 2 days, properties of FAs do not change much. This also suggests that with time, FAs change their main function from the transmission of strong propulsive forces to a more passive function of providing adhesion sites for 


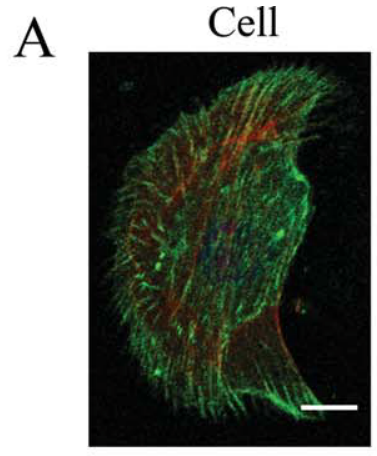

Fibroblast
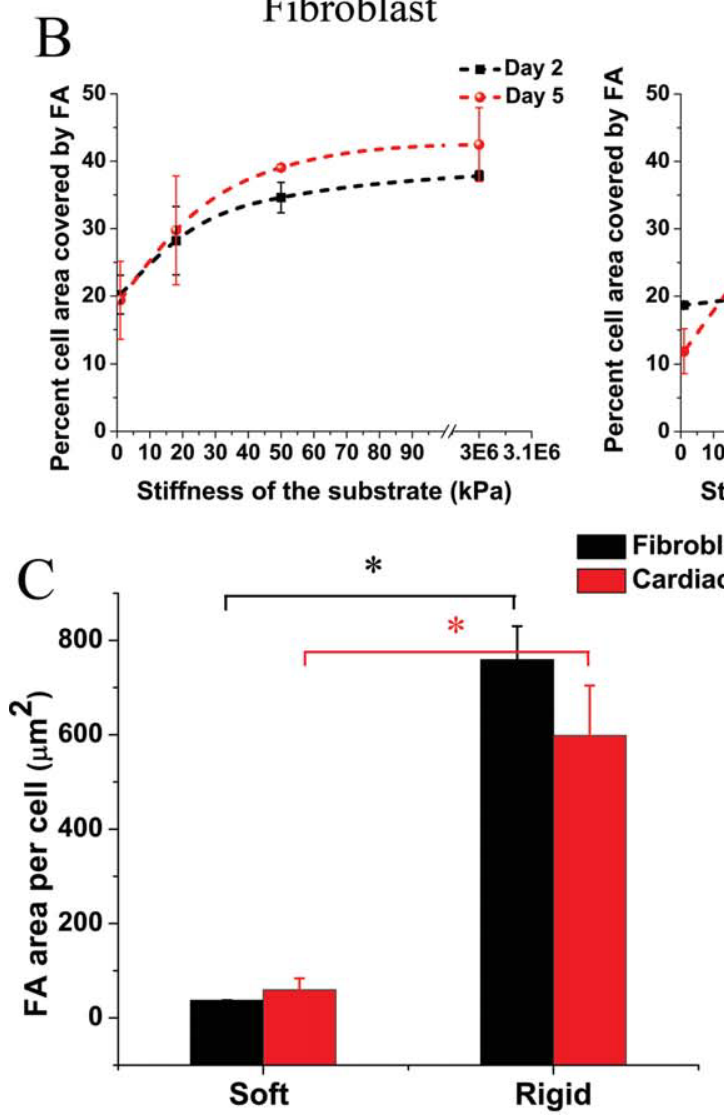

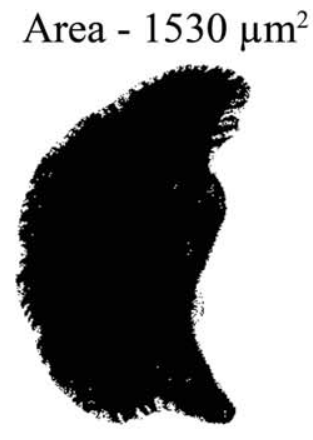

Cardiac cell

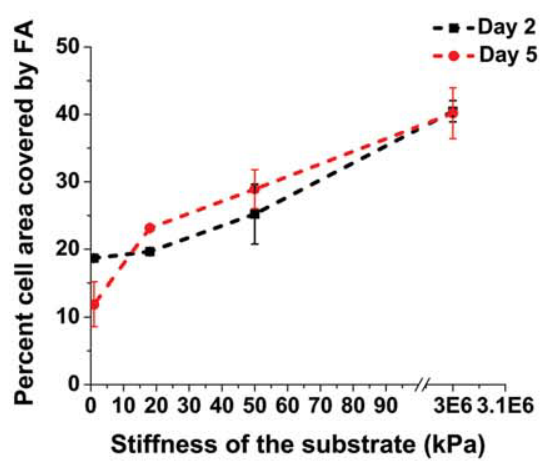

Fibroblast

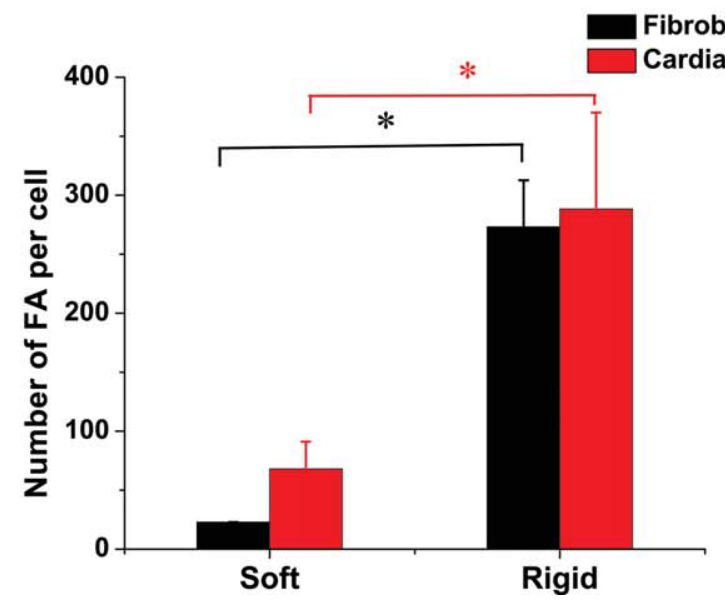

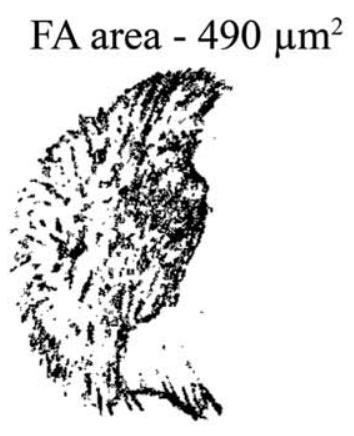

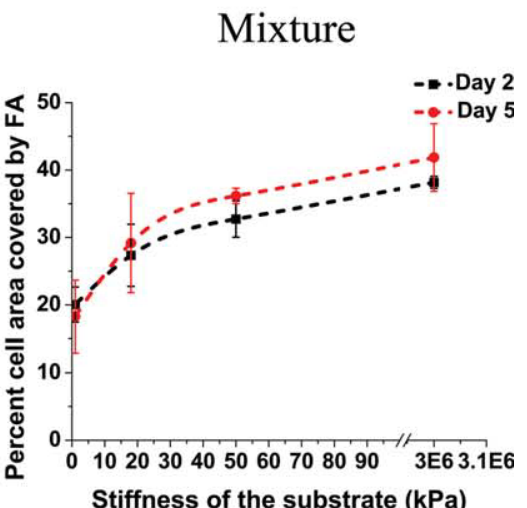

Fibroblast diac cell

FIGURE 3. Quantification of FAs (A). The process used for quantification of FAs. The FAs were masked and their percentage in the cell was calculated as a ratio of the total area. (B) Quantification of FA area for fibroblasts, cardiac cells, and their mixture for the $1 \mathrm{kPa}, 18 \mathrm{kPa}, 50 \mathrm{kPa} P A$ gels, and TC dishes. The percentage of FAs for the mixture was computed as a weighted average from fibroblasts and cardiac cells. (C) Quantification of the number of FA/cell $(p<0.05)$ and the average FA area/cell $(p<0.05)$ for the soft $(1 \mathrm{kPa})$ and the rigid (TC dish) substrates on day 2 . Asterisks indicate statistical significance between the soft 1-kPa PA gel and the rigid TC dish. At $p<0.05$, the means are statistically different. Data show are the mean $\pm \operatorname{SEM}(n=20)$. Scale bar: $10 \mu \mathrm{m}$.

the maintenance of a well spread cell morphology. ${ }^{37}$ Fibroblasts were identified from cardiac cells based on the sarcomeric striation patterns seen on the cardiac cells. Figure $3(\mathrm{C})$ shows that the number of FAs per cell $(p<0.05)$ and the FA area per cell $(p<0.05)$ are much higher on the rigid (TC) substrate compared with the soft $(1 \mathrm{kPa})$ gel substrate. The means of all these parameters are statistically different for the rigid and soft gels at $p<0.05$.

Figure 4(A) shows the actin cytoskeleton on the soft 1$\mathrm{kPa}$ PA gel. It can be seen that even though there are striations visible on the cells on this soft gel, which is a hallmark 

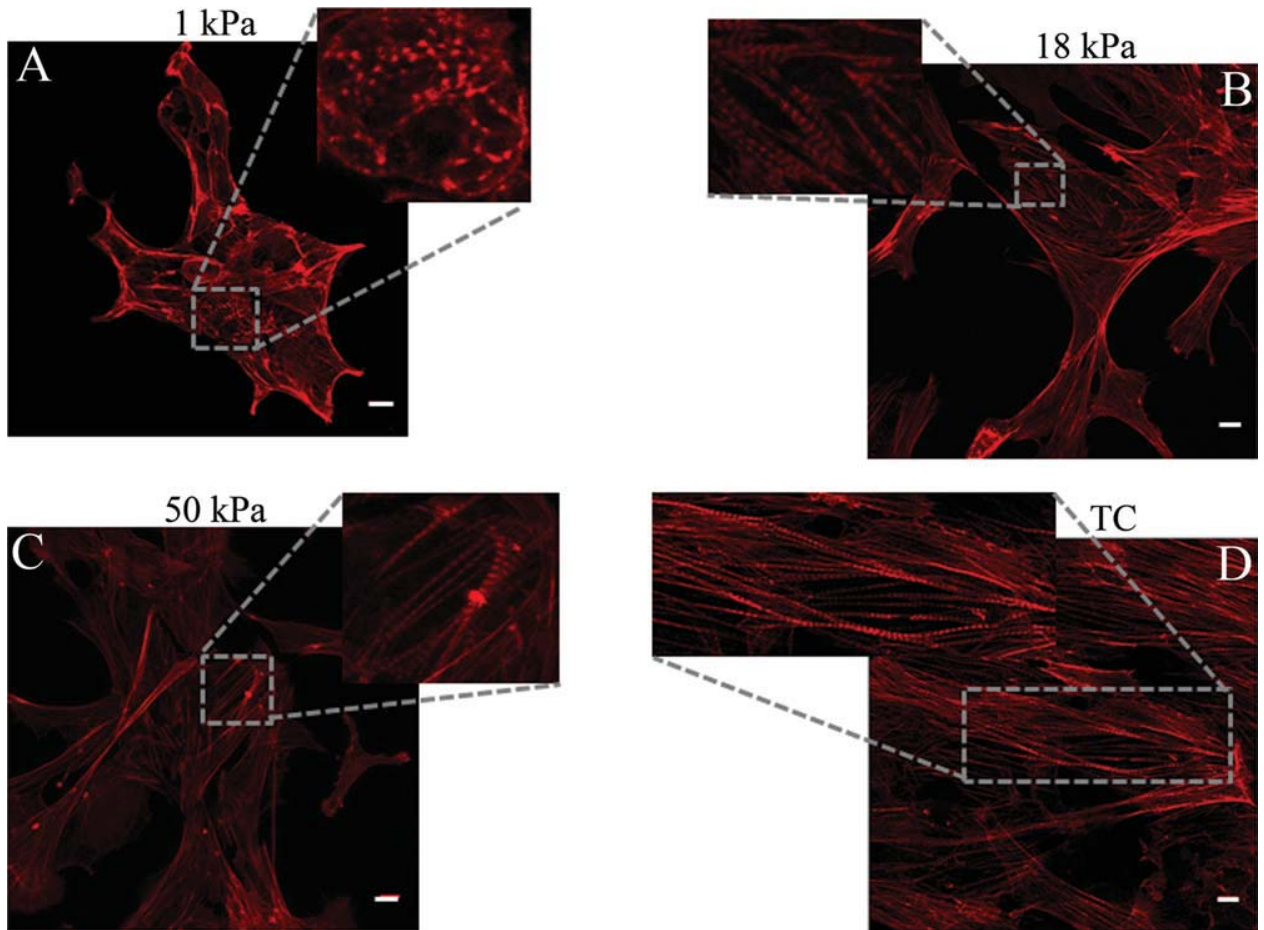

FIGURE 4. Cytoskeletal structure of the cells on the substrates. Single stack confocal images for the cells on the (A) $1 \mathrm{kPa}$, (B) $18 \mathrm{kPa}$, (C) $50 \mathrm{kPa}$ PA gels, and (D) the TC dish. The insets in the figures show a zoomed view of the sarcomeric striations. Scale bar: $10 \mu \mathrm{m}$.

MEMS sensor was $\sim 0.25 \mathrm{nN}$. The MEMS force sensor has two beams, a fixed (reference) beam and a free (measurement) beam, which touches the cell cluster as seen in Figure 5(A). The MEMS senor was kept in a solution of $70 \%$ ethanol for $2 \mathrm{~h}$ under UV light to sterilize the sensor before starting the measurements. The MEMS sensor was cleaned by sterilized DI water multiple times before experiments to remove any ethanol residue or micro-physisorptions on device. To eliminate the external mechanical indentation, the forward movement of the beam was stopped right after contacting the cell body (see Fig. S2). The cyclic contraction force, $F$, was quantitatively obtained from, $F=k \Delta x$, where, $k=48.96 \mathrm{nN} / \mu \mathrm{m}$, the spring constant of the force sensor, and $\Delta x$ is the deformation of the sensor spring. Figure 5(B) shows the phase-contrast image of the MEMS probe in contact with the $18-\mathrm{kPa}$ gel, with the sensor probe contacting the cell body along the direction of contraction.

Figure 5(C) shows the force profile of the cardiac cell on both the $18-\mathrm{kPa}$ gel and the TC substrate as a function of time on the fifth day. The duration for one contractionrelaxation cycle on both substrates was about 0.6-0.8 s. Figure 5(D) shows a bar graph of the force measured by the MEMS probe as a function of stiffness on both the second and fifth days. The force exerted on the MEMS sensor by the cells cultured on TC dish $\left(F_{5 \text { th day }}=71.30 \pm 6.38 \mathrm{nN}\right)$ was statistically higher than that by the cells cultured on the soft $18-\mathrm{kPa}$ gel $\left(F_{5 \text { th day }}=30.16 \pm 3.83 \mathrm{nN}\right)$ at $p<$ 0.05 , and the same trend was seen for FAs as well. The isometric contractile stress that cardiac myocytes applied on their fibroblast surrounding can be estimated by consider- ing that the beating force measured by the force sensor is only sampled from a portion of the cell membrane (contact area of $\sim 25 \mu^{2}$ ). The value of the stress on the TC dish was calculated to be $3.2 \mathrm{nN} / \mu^{2}$, whereas that on the 18 $\mathrm{kPa}$ gel was $2.7 \mathrm{nN} / \mu^{2}$ on the second day. These values are close to the contractile stress of neonatal rat cardiac myocytes $\left(5.5 \pm 2 \mathrm{nN} / \mu \mathrm{m}^{2}\right)$, which has been reported elsewhere. $^{40}$

\section{DISCUSSION}

ECCMs were seeded on laminin-coated PA gels with a range of stiffness from 1 to $50 \mathrm{kPa}$ and their beating rate was monitored as a function of time over 5 days. It was found that the cells beat with the highest frequency on the substrate, which mimics the stiffness of the myocardium $(\sim 18$ $\mathrm{kPa}$ ) as previously shown. ${ }^{41}$ However, in our study, the cells had the highest beating rate on the $18-\mathrm{kPa} \mathrm{PA}$ gel only during the first few days. After that, the beating rate for the cells on all the substrates converged to a narrow band between 60 and 80 beats per minute. A plausible mechanism for this is that during the initial days, when the cells are seeded on the substrate, no cell-cell interactions are possible because a confluent cell sheet is not formed. However, by the second day, cells started forming cell-cell junctions, and a cell network was seen forming on the stiffer substrates (18 kPa, $50 \mathrm{kPa}$, and TC). Fibroblasts started proliferating between neighboring cardiac cells, which linked them together. It has been shown that the beating rates of cardiac cells within $300 \mu \mathrm{m}$ can be synchronized through the fibroblast connection, ${ }^{42}$ and a similar phenomenon was 

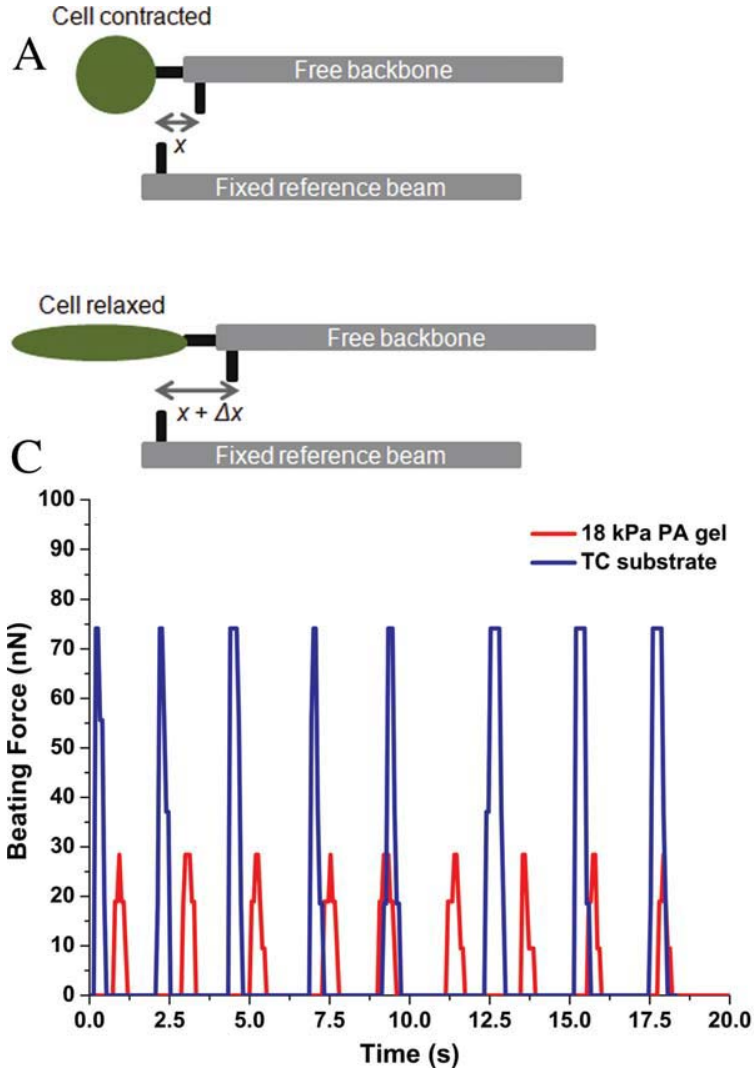

B

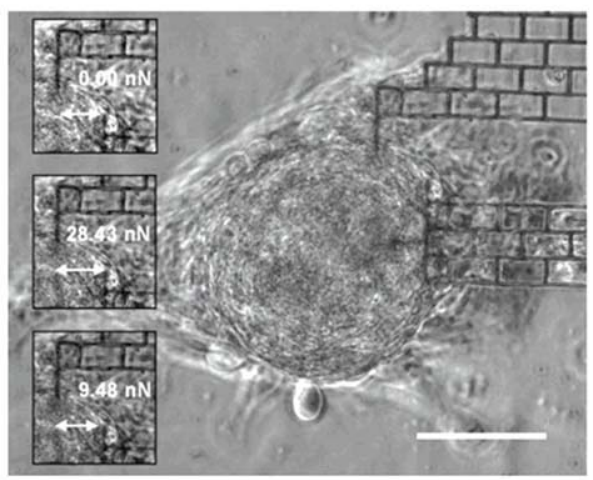

$\mathrm{D}$

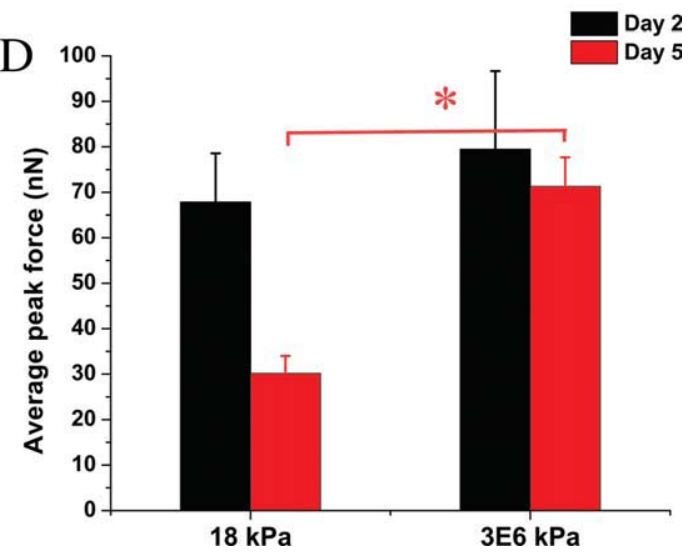

FIGURE 5. MEMS force measurement on the substrates (A). The principle of operation (B). Phase-contrast image showing a cardiac cell aggregate with MEMS force sensor attaching from right side to measure the beating force during contraction-relaxation cycle. Cell is on 18-kPa gel on the fifth day of culture. (C) The force profile of the cardiac cell cluster sensed by the MEMS force sensor as a function of time on the 18-kPa PA gel and the TC dish. (D) Bar graphs showing the average peak force exerted by the cardiac cell cluster on the 18-kPa PA gel and the TC dish on the second and fifth day. Asterisks indicate statistical significance between the average peak force on day 5 between $18 \mathrm{kPa}$ PA gel and TC dish (3E6 kPa). At $p<0.05$, the means are statistically different. Data shown are the mean \pm standard deviation (SD; $n=3$ ). Scale bar: $50 \mu \mathrm{m}$.

observed during our experiment when two cardiac cells were connected via a fibroblast (see Supporting Information Movie S1). It is known that fibroblasts do not extend their processes on soft substrates $(1 \mathrm{kPa})$ and remain rounded. ${ }^{36}$ So how do the cells on the soft $1-\mathrm{kPa}$ gel beat around the same frequency as well? One of the reasons could be that the beating frequency of the cells reverts to a natural beating frequency that is inherent to the cell as seen in previous studies. ${ }^{43}$ Another possible reason could be that when the cells are close to each other, the substrate itself may serve as a mechanical link between the substrates. Contraction of a cell during beating results in stretching of the nearby substrate, which in turn stretches the neighboring cells' membrane and a cascade of signaling mechanisms start in this neighboring cell (see Supporting Information Movie S2). It would be interesting to see if this convergence phenomenon is still seen on the stiffer substrates when an enrichment of the cell mixture is done to limit the number of fibroblasts by pre-plating this cell mixture before seeding them on the different substrates.

Previous reports have shown that optimum actomyosin striations were seen on substrates that mimic the stiffness of the myocardium. ${ }^{5,44,45}$ However, even on the rigid TC sub- strate, we were able to see highly aligned sarcomeric patterns. This could be because cell-cell contacts might have a similar effect as cell-on-gel effect when a cellular network is formed. This was also seen in another study when endothelial cells had indistinguishable morphology on the stiff and the soft substrates when they reached confluence. ${ }^{36}$ Furthermore, fibroblasts, which generally cannot extend their processes and remain rounded on the soft gels, are seen to have a well spread morphology when cell-cell contacts are established. ${ }^{36}$ Myofibrillogenesis involves the precise stacking of multiple linear array of units into a structured sarcomere. ${ }^{46}$ It starts with the formation of pre-myofibrils at the periphery of spreading cardiomyocytes and get bundled into mature myofibrils over time. ${ }^{47}$ However, because the soft 1$\mathrm{kPa}$ substrate cannot provide enough actomyosin-based contractions, formation of aligned sarcomeric structure is not seen on this substrate. On the other three substrates, enough tension is developed from the mature FAs that the entire process of myofibrillogenesis is possible.

It has been well known that most normal cells in cell culture are anchorage dependent. ${ }^{31}$ FAs are the sites of attachment from the intracellular cytoskeleton via integrin receptors to the extracellular matrix. Formation and development 
of FAs require generation of isometric tension by movement of myosin II motors on the actin filaments. Becuase cells on the soft substrate (1-kPa PA gel) cannot develop this isometric tension by the actomyosin contractions, few FAs are formed on this substrate. Furthermore, the FAs that are formed on this substrate are mostly immature because aggregation of integrins is not possible on the soft substrate because of the reduced actomyosin contractions. As a result, cells on this substrate resemble tissue-like morphology where a lot of cells are present in the aggregate. On the other hand, cells on stiffer substrates are able to generate isometric tension by actomyosin contractions leading to their maturation. It should be noted that the morphology of cells on the $50-\mathrm{kPa}$ PA gel is similar to that on the rigid TC dish. Furthermore, as seen in Figure 3(B), there is a small increase in the percentage of FAs from the $50-\mathrm{kPa}$ substrate to the TC substrate, and the graph seems to reach a steady value. This means that after a certain threshold, the stiffness of the substrate is no longer the dominant factor in the formation of FAs. Any further increase in the stiffness of the substrate after the threshold stiffness will only lead to small changes in the overall morphology of the cells and growth of FAs. However, for the cells to perform their functions optimally, a stiffness matching between the cell and the substrate is desired. ${ }^{41}$

A novel MEMS-based force sensor was used to measure the force of contraction of the cardiac cell cluster on the 18$\mathrm{kPa}$ PA gel substrates and the TC dish. It was found that the force of contraction as seen by the MEMS sensor was larger on the TC dish than on the 18-kPa gel. This higher force on the rigid TC dish could be because of the formation of higher percentage of FA on this substrate compared with the $18 \mathrm{-kPa}$ gel as shown in Figure 3(B). It should be noted that the force exerted on the MEMS sensor might not be the total beating force of the cardiac cell cluster. Part of the force is lost in the displacement of the PA gels when the cell is contracting. In addition, the size of the cluster chosen might have an important effect on the force seen by the MEMS sensor. The force seen by the MEMS probe on the rigid TC dish on days 2 and 5 was not different as shown in Figure 5(D). Also, the percentage of FAs remained similar during this period on the TC dish as shown in Figure 3(B). The sensor is based on the principle of Hookean mechanics, $F=k \Delta x$; therefore, if the placement of the sensor is not optimum for sensing the maximum contraction, a big difference in the force is possible. A shortcoming for the sensor is that it is dependent on the size of the cluster and the location of the sensor with respect to the cluster. If both these parameters are not optimized, an error in the force measurement is possible. Although these are some of the uncertainties associated with the MEMS sensor, it can be used effectively to give a firsthand approximation for the force of a beating cardiac cell/cluster.

\section{CONCLUSIONS}

We have shown that ECCMs beat with the highest beating rate on the substrates with tissue-like stiffness only during the first few days after plating, and later the beating rate becomes independent of the stiffness. However, the cells form bigger and higher number of FAs with an increase in the stiffness of the substrate resulting in higher forces on the stiffer substrates. Furthermore, highly aligned sarcomeric striations are only seen on the stiffer substrates, whereas the soft substrate shows nonaligned striations. Given the profound effect of stiffness of the substrate on the behavior of cardiac cells, future scientists and engineers must take into account the physical parameters of the materials, such as rigidity, in designing the next generation of biomaterials.

\section{ACKNOWLEDGMENTS}

The authors thank Judy Grimmer at Prof. Riyi Shi's lab at Purdue University for assistance in the isolation of the embryonic chicken myocytes, Dr. Larry Millet, Dr. Adarsh Radadia, and Vincent Chan for helpful discussions. The authors also thank the staff of the Bionanotechnology Laboratory at Micro and Nanotechnology Laboratory (MNTL) and Dr. Mayandi Sivaguru of the Institute of Genomic Biology (IGB) at UIUC.

\section{REFERENCES}

1. Engler AJ, Sen S, Sweeney HL, Discher DE. Matrix elasticity directs stem cell lineage specification. Cell 2006;126:677-689.

2. Flanagan LA, Ju YE, Marg B, Osterfield M, Janmey PA. Neurite branching on deformable substrates. Neuroreport 2002;13: 2411-2415.

3. Solon J, Levental I, Sengupta K, Georges PC, Janmey PA. Fibroblast adaptation and stiffness matching to soft elastic substrates. Biophys J 2007;93:4453-4461.

4. Lo $\mathrm{CM}$, Wang H-B, Dembo M, Wang YL. Cell movement is guided by the rigidity of the substrate. Biophys J 2000;79:144-152.

5. Engler AJ, Griffin MA, Sen S, Bönnemann CG, Sweeney HL, Discher DE. Myotubes differentiate optimally on substrates with tissue-like stiffness. J Cell Biol 2004;166:877-887.

6. Wang HB, Dembo M, Wang YL. Substrate flexibility regulates growth and apoptosis of normal but not transformed cells. Am J Physiol Cell Physiol 2000;279:C1345-C1350.

7. Lammerding J, Kamm RD, Lee RT. Mechanotransduction in cardiac myocytes. Anal N Y Acad Sci 2004;1015:53-70.

8. Discher DE, Janmey $\mathrm{P}$, Wang YL. Tissue cells feel and respond to the stiffness of their substrate. Science 2005;310:1139-1143.

9. Engler AJ, Rehfeldt F, Sen S, Discher DE. Microtissue elasticity: Measurements by atomic force microscopy and its influence on cell differentiation. Methods Cell Biol 2007;83:521-545.

10. Berry MF, Engler AJ, Woo YJ, Pirolli TJ, Bish LT, Jayasankar V, Morine KJ, Gardner TJ, Discher DE, Sweeney HL. Mesenchymal stem cell injection after myocardial infarction improves myocardial compliance. Am J Physiol Heart Circ Physiol 2006;290: H2196-H2203.

11. Litwin S, Litwin C, Raya T, Warner A, Goldman S. Contractility and stiffness of noninfarcted myocardium after coronary ligation in rats. Effects of chronic angiotensin converting enzyme inhibition. Circulation 1991;83:1028-1037.

12. Wang $Y L$, Pelham RJ. Preparation of a flexible porous polyacrylamide substrate for mechanical studies of cultured cells. Methods Enzymol 1998;298:489-496.

13. Damljanovic V, Lagerholm BC, Jacobson K. Bulk and micropatterned conjugation of extracellular matrix proteins to characterized polyacrylamide substrates for cell mechanotransduction assays. BioTechniques 2005;39:847-851.

14. Cukierman E, Pankov R, Stevens DR, Yamada KM. Taking cell-matrix adhesions to the third dimension. Science 2001;294: 1708-1712.

15. Engler AJ, Bacakova L, Newman C, Hategan A, Griffin M, Discher D. Substrate compliance versus ligand density in cell on gel responses. Biophys J 2004;86:617-628.

16. Gefen A, Margulies SS. Are in vivo and in situ brain tissues mechanically similar? J Biomech 2004;37:1339-1352. 
17. Hertz HR. Uber die Beruhrung fester elastischer Korper. J.f.d. Reine u. Angewandte Math 1882;92:156-171.

18. Radmacher M. Measuring the elastic properties of biological samples with the AFM. IEEE Eng Med Biol Magn 1997;16:47-57.

19. Engler AJ, Richert L, Wong JY, Picart C, Discher DE. Surface probe measurements of the elasticity of sectioned tissue, thin gels and polyelectrolyte multilayer films: Correlations between substrate stiffness and cell adhesion. Surf Sci 2004;570:142-154.

20. Hamburger V, Hamilton HL. A series of normal stages in the development of the chick embryo. Dev Dyn 1992;195:231-272.

21. Abramoff MD, Magelhaes $P$, Ram SJ. Image processing with ImageJ. Biophotonics Int 2004;11:36-42.

22. Marquez M, Fernandez-Tome MD, Favale N, Pescio L, Sterin-Speziale NB. Bradykinin modulates focal adhesions and induces stress fiber remodeling in renal papillary collecting duct cells. Am J Physiol Renal Physiol 2008;294:F603-F613.

23. Cavalcanti-Adam EA, Micoulet A, Blummel J, Auernheimer J, Kessler $\mathrm{H}$, Spatz JP. Lateral spacing of integrin ligands influences cell spreading and focal adhesion assembly. Eur J Cell Biol 2006; 85:219-224.

24. Frey MT, Tsai IY, Russell TP, Hanks SK, Wang YL. Cellular responses to substrate topography: Role of myosin II and focal adhesion kinase. Biophys J 2006;90:3774-3782.

25. Katz BZ, Zamir E, Bershadsky A, Kam Z, Yamada KM, Geiger B. Physical state of the extracellular matrix regulates the structure and molecular composition of cell-matrix adhesions. Mol Biol Cell 2000;11:1047-1060.

26. Guo WH, Frey MT, Burnham NA, Wang YL. Substrate rigidity regulates the formation and maintenance of tissues. Biophys J 2006 90:2213-2220.

27. Lee SW, Otto JJ. Vinculin and talin: Kinetics of entry and exit from the cytoskeletal pool. Cell Motil Cytoskeleton 1997;36: 101-111.

28. Geiger B, Bershadsky A, Pankov R, Yamada KM. Transmembrane extracellular matrix-cytoskeleton crosstalk. Nat Rev Mol Cell Biol 2001;2:793-805.

29. Saunders RM, Holt MR, Jennings $L$, Sutton DH, Barsukov IL, Bobkov A, Liddington RC, Adamson EA, Dunn GA, Critchley DR. Role of vinculin in regulating focal adhesion turnover. Eur $\mathrm{J}$ Cell Biol 2006;85:487-500.

30. Humphries JD, Wang P, Streuli C, Geiger B, Humphries MJ, Ballestrem C. Vinculin controls focal adhesion formation by direct interactions with talin and actin. J Cell Biol 2007;179:1043-1057.

31. Burridge K, Chrzanowska-Wodnicka M. Focal adhesions, contractility, and signaling. Annu Rev Cell Dev Biol 1996;12:463-518.

32. Chrzanowska-Wodnicka M, Burridge K. Rho-stimulated contractility drives the formation of stress fibers and focal adhesions. J Cell Biol 1996;133:1403-1415.
33. Pelham RJ, Wang YL. Cell locomotion and focal adhesions are regulated by substrate flexibility. Proc Natl Acad Sci USA 1997;94: 13661-13665.

34. Zaidel BR, Ballestrem C, Kam Z, Geiger B. Early molecular events in the assembly of matrix adhesions at the leading edge of migrating cells. J Cell Sci 2003;116:4605-4613.

35. Saez A, Buguin A, Silberzan P, Ladoux B. Is the mechanical activity of epithelial cells controlled by deformations or forces?. Biophys J 2005;89:L52-L54.

36. Yeung $T$, Georges $P C$, Flanagan LA, Marg B, Ortiz M, Funaki M, Zahir N, Ming WY, Weaver V, Janmey PA. Effects of substrate stiffness on cell morphology, cytoskeletal structure, and adhesion. Cell Motil Cytoskeleton 2005;60:24-34.

37. Beningo KA, Dembo M, Kaverina I, Small JV, Wang YL. Nascent focal adhesions are responsible for the generation of strong propulsive forces in migrating fibroblasts. J Cell Biol 2001;153: 881-887.

38. Yang S, Saif MT. Micromachined force sensors for the study of cell mechanics. Rev Sci Instrum 2005;76:044301-044301-8.

39. Yang S, Saif MT. Force response and actin remodeling (agglomeration) in fibroblasts due to lateral indentation. Acta Biomater 2007;3:77-87.

40. Balaban NQ, Schwarz US, Riveline D, Goichberg P, Tzur G, Sabanay I, Mahalu D, Safran S, Bershadsky A, Addadi L, Geiger B. Force and focal adhesion assembly: A close relationship studied using elastic micropatterned substrates. Nat Cell Biol 2001;3: 466-472.

41. Engler AJ, Carag-Krieger C, Johnson CP, Raab M, Tang HY, Speicher DW, Sanger JW, Sanger JM, Discher DE. Embryonic cardiomyocytes beat best on a matrix with heart-like elasticity: Scarlike rigidity inhibits beating. J Cell Sci 2008;121:3794-3802.

42. Kohl P, Camelliti P, Burton FL, Smith GL. Electrical coupling of fibroblasts and myocytes: Relevance for cardiac propagation. J Electrocardiol 2005;38:45-50.

43. Harary I, Farley B. In vitro studies on single beating rat heart cells: Growth and organization. Exp Cell Res 1963;29:451-465.

44. Jacot JG, McCulloch AD, Omens JH. Substrate stiffness affects the functional maturation of neonatal rat ventricular myocytes. Biophys J 2008;95:3479-3487.

45. Bhana B, lyer RK, Chen WL, Zhao RG, Sider KL, Likhitpanichkul M, Simmons CA, Radisic M. Influence of substrate stiffness on the phenotype of heart cells. Biotechnol Bioeng 2010:1148-1160.

46. Dabiri GA, Turnacioglu KK, Sanger JM, Sanger JW. Myofibrillogenesis visualized in living embryonic cardiomyocytes. Proc Natl Acad Sci USA 1997;94:9493-9498.

47. Dlugosz AA, Antin PB, Nachmias VT, Holtzer $H$. The relationship between stress fiber-like structures and nascent myofibrils in cultured cardiac myocytes. J Cell Biol 1984;99:2268-2278. 